\title{
2021 Undergraduate Big Data Challenge: Infodemiology for the future of digital and public health
}

$10.17975 /$ sfi-2021-003

STEM Fellowship's Undergraduate Big Data Challenge (UnBDC) is an inquiry-driven and experiential learning program that invites students from across the country to strengthen their problem-solving and critical thinking skills while gaining familiarity with the fundamentals of data science. By allowing students to undertake independent research projects that tackle real-world public health and bioinformatics problems, the BDC fosters scientific inquiry and prompts new and innovative ideas.

This year, we invited students to investigate the theme of Infodemiology for the Future of Digital and Public Health. It allowed students to explore practical applications and insights of infodemiology to discover breakthrough connections in Digital and Public Health using open social, demographic, and health data. Students explored many topics, ranging from using Twitter machine learning for fighting the COVID-19 infodemic, to the sentiment comparison between real and fake COVID-19 news articles.

We developed in-depth learning modules designed to lead the student from zero-knowledge to an elementary working proficiency in data science. The students learn a broad range of data analytics tools, methods and programming languages which are useful for uncovering hidden patterns, trends in structured and unstructured data. Some of the skills the students learnt and used includes Data Visualization, Classification, Statistics and Data Handling, Overleaf etc.

On behalf of STEM Fellowship, we extend our sincere congratulations to all students who participated in the challenge, and wish them the best for their future endeavours. It has been a privilege for us to witness the analytical capabilities of the next generation of students firsthand, and we are certain all entrants will continue to demonstrate excellence in their respective careers.

Anish Verma

Chief Data Officer
Dr. Sacha Noukhovitch

Founder, President and Editor-in-Chief

\section{Acknowledgments}

This event would not have been possible without the generous support of Hoffman La Roche, JMIR Publications, Canadian Science Publishing, Digital Science, Overleaf and SAS Canada. Special thanks to all our experts, judges and team mentors who reviewed the participating teams' submissions and evaluated the finalists. We are also grateful to our volunteers who made this program successful.

\section{Disclaimer}

These abstracts are provided for all student teams that have submitted to UnBDC 2021. The STEM Fellowship Journal editorial board has made every effort to ensure proof and English editing of these abstracts in a limited amount of time, and neither organization as a whole or any of its volunteer members can be held accountable for inaccuracies that may have occurred in the abstract publication. Abstracts are published in alphabetical order per school names of the participating teams. 


\section{International Sentiment Tracker for December,} 2020

\section{Randa Hassan, Sara Hassan, Amulya Kaianathb- hatta, and Seneli Seneviratne}

This study assessed the correlation between emotional sentiment and country of origin for tweets gathered in December of 2020. The purpose of this study was to evaluate the extent that the national context affected the national and, consequently, international public trust in governments, national healthcare systems, health guidelines, and the vaccine rollout process. Literature review has shown that more socioeconomically advantaged countries generally provided better access to public healthcare and had better access to vaccine doses in December of 2020. As such, it is hypothesized that more socioeconomically-advantaged countries will have more positive sentiments towards the COVID-19 pandemic, while less socioeconomically-advantaged countries will have more negative sentiments towards the pandemic. Opensource datasets [4] of multinational and multilingual tweets containing sentiment analysis data relating to Covid-19 from December, 2020 were used to test this hypothesis. A Naive Bayes algorithm was used to train the dataset and to determine the correlation between the sentiments of tweets and national context. Results were inconclusive as to the correlation between country of origin and sentiment. This study demonstrates the ease by which governments and institutions can gauge public trust and compliance with health guidelines. This will allow governments to potentially improve their own healthcare systems and public outreach, thus improving reliability and reducing healthcare disparities.

Predicting Falsehood of a Tweet Concerning COVID-19 Using Location, Socioeconomic Data, and Tweet Sentiment

\section{Danish Baig, KaHo Wong, and Sabina Henry}

During the COVID-19 pandemic, the spread of false information has been detrimental to the public. Efforts have been spent on identifying false information and preventing its propaga- tion. In this work, we attempted to predict the falsehood of a tweet concerning COVID-19 by analyzing the location, socioeconomic data, and text sentiment associated with the tweet. The main resource used for this work was the CoAID data set which contains tweet IDs for the original tweet and their replies, pre-labelled as 'Real' or 'Fake' COVID-19 info. The Hydrator software was used to crawl the user location and content of the tweet from tweet IDs. Socioeconomic data associated with the user's location was matched with the tweet ID data set. A set of logistic regression models on both original tweets and replies was created by mapping various combination of indicators to the pre-labelled data set. From these models, a mix of socioeconomic variables and sentiment data trained on the tweets resulted in better accuracy when predicting the tweet's factuality, compared to models using other combinations of indicators trained on the reply data set. Future work should consider analyzing other models, as well as adopting higher geographical resolution data for more accurate predictions.

\section{An Analysis of the COVID-19 Infodemic: Influence of the Frequency of Keywords and Type of Words on the Validity of Tweets and Articles}

\section{James Beninger, Brenda Zou, and Tian Hui Zhao}

Patterns of language use are arguably present in all different social situations, and the ability to identify such patterns is essential for large scale analysis of social media. If future infodemics are to be avoided, then we must develop tools to identify misinformation en masse, and facilitate the spread of accurate information. Here, we use pattern recognition and categorization techniques on large datasets of tweets and news article titles to mimic the early stages of machine learning, and to identify if there are any patterns simple enough for algorithmic artificial intelligence to identify for the purpose of providing solutions for the COVID-19 infodemic.

\section{Pipeline for identifying vaccine-specific info- demic insights from Youtube and Reddit and leveraging NLP deep learning models to predict misinformation}

Nikhil Saini, Nirupama Tamvada, and Mansi Patel

Amidst the continuing COVID-19 pandemic, the administration of the SARS-CoV-2 vaccines is imperative 
to achieve herd immunity and curtail further spread of the virus. However, the COVID-19 infodemic, pertaining to the dissemination of both reliable and dubious information on news and social network platforms, is profoundly shaping people's attitudes towards COVID-19 vaccines, thus, resulting in vaccine hesitancy. Accordingly, public sentiment for COVID-19 vaccines in relation to pertinent vaccine-related events (blood clots with the AstraZeneca vaccine in rare cases, efficacy of the vaccines to the variants, Pfizer vaccine au- thorization for children, and heart inflammation in young Pfizer vaccine patients) was assessed. Data from YouTube and Reddit was assessed and used in a comparative manner. Sentiment analysis, time-series analysis, topic modelling, the reliability of information commonly shared on Reddit, and sources of community misinformation identification were carried out to identify events, misinformation, sources, and topics associated with COVID-19 vaccine hesitancy. Our findings indicated platform-based differences in public sentiments, specific subreddits promoting misinformation on Reddit and image-based sources and memes being a common source of information. Subsequently, a misinformation classifier specifically for Reddit data, capable of handling such images was trained on recent labelled data from identified subreddits and developed to classify reliable and unreliable information using two different classification methodologies: Recurrent Neural Network and XGBoost. Our findings and our classifier hold significant applications to allow for curbing the spread of misinformation on discussion-based platforms such as Reddit, as well as in tailoring public health campaigns in combatting vaccine hesitancy.

\section{Characterization and Detection of Misinforma- tion to Inform Healthcare Policies}

Amin Abdolkhani, Umar Ali, Hamza Rasheed, and Johnny Zhao

The rampant spread of misinformation regarding COVID-19 is abundant on social media platforms. There is a clear need to investigate the characteristics of misinformation as it relates to healthcare in order to create effective policies. In this paper, we explore the primary characteristics of fake Twitter posts by examining the frequency, length, and vocabulary of their words used. Additionally, we use a logistic regression model, reaching an accuracy of $93.6 \%$, to verify the veracity of Twitter posts relating to COVID-19. We propose a comprehensive list of guidelines for healthcare workers to consider when consulting patients; the aim of this list is to address the immediate characteristics of social media misinformation. By informing patients of the characteristics of social media misinformation, we hope to mitigate the perception of fake COVID-19 news as fact.

\section{Utilizing BERT Model and K-Means Clustering for Geo-mapping the COVID-19 Misinformation Vul- nerability in Canadian Regions Based on Social Factors}

\section{Maliha Lodl, Afnan Rahman, Fatima Sadia, and Farhat Ullah}

COVID-19 has increased the vulnerability of misinformation in communities around the world. The vulnerability of the misinformation will be categorized through age, socioeconomic status, trust in social media, political polarization and traditional news viewership. The research team will investigate whether certain communities in Canada are more vulnerable to COVID-19 related misinformation, compared to others. Using BERT, the CoAID: COVID-19 Healthcare Misinformation Dataset curated by Harvard University, and different NLP methods, we will identify the COVID-19 related tweets and the validity of the information. The research will also regionally group these tweets by severity of misinformation using k-means clustering. Expected results will allow us to compare each regions' age, economic status, and the literacy rate to the clusters themselves to find which clusters are the most vulnerable to misinformation. Our team hypothesizes that geographical clusters with older residents, lower literacy rates and low socioeconomic status will be more vulnerable to COVID-19 misinformation. The most misinformed clusters can be educated through awareness campaigns, workshops can be set up within their local community, which will prevent the spread of health misinformation.

\section{Introducing ADMIT : A First Step in Uncovering FDA's Deceit}

Jérémie Babeu, Zoé Benoit, Andréanne Boulanger, and Antoine Turcotte 
Medical implants are now an integral part of public health in the United States. However, the last few years have brought several controversies regarding their regulation, considering the large number of deleterious effects reported by patients. Some authors even denounce the lack of rigor and transparency in the process of marketing medical implants as well as in the reporting of data. This article aims to address these shortcomings by introducing the American Database for Medical Implant Transparency (ADMIT), a database integrating data from multiple governmental sources to characterize the entire process by which implantable medical implants are approved by the FDA. After exploring the potential of ADMIT through preliminary analyses, it will be published on an open access platform. Ultimately, it is hoped that this initiative will stimulate research into the real negative effects of gaps in medical implant regulation while providing an informative tool for patients seeking information about them.

\section{The Plebeian Algorithm A Democratic Approach to Censorship and Moderation}

\section{Benjamin D. Fedoruk, Harrison S. Nelson, Kai A. Fucile Ladouceur, and Russell M. Frost}

The infodemic created by the COVID-19 pandemic has created several societal issues problems, including a rise in distrust between the public and health experts and even a refusal of some to accept vaccination, with some sources suggesting that 1 in 4 American will refuse the vaccine [1]. This social concern can be traced to the level of digitization today - particularly in the form of social media. As social media was the most significant contributing factor to the spread of misinformation, the team decided to examine infodemiology across various text- based platforms (Twitter, 4chan, Reddit, Parler) by utilizing a sentiment analysis to compare general posts with key terms flagged as misinformation (all of which concern COVID-19), to determine their verity. In gathering the datasets, both APIs and also pre-existing data compiled by standard scientific third parties were used. It was found that in some cases, misinforming posts can have up to $92.5 \%$ more negative sentiment skew compared to accurate posts. From this, the novel Plebeian Algorithm is proposed, which utilizes sentiment analysis and post popularity as a metric to flag a post as misinformation. This algorithm diverges from that of the status quo, as the Plebeian Algorithm uses a democratic process to detect and remove misinformation. A method was constructed in which content deemed misinformation to be removed from the platform is determined by a randomly selected jury of anonymous users. This not only prevents these types of "infodemics", but also guarantees a more democratic way of using social media that is beneficial for restoring the social distrust of medical experts and encouraging the public's evidence-informed decision making.

\section{Bird Watch now Informs users about COVID-19 Tweet Corrections}

\section{Benny Guan, Zahra Niazi, Deep Patel, William Young-Hong}

The COVID-19 pandemic has resulted in the mass spread of misinformation worldwide which has global impacts on public health. This situation has been declared as an infodemic and it has significant effects on the management of public health responses to disease control. An infodemic involves intentional widespread distribution of misinformation, effectively hindering public health's ability to manage and control the disease. As such, the public is unsure about what information to trust, and what information to be wary of. Specifically, misinformation can lead to the loss of life when public health guidelines are not trusted resulting in diagnostic testing going unused, and vaccinations not reaching their intended audience. To address this issue, it is vital to limit and stop the initial spread of fake news. One major vehicle of transmission for misinformation is social media. For example, Twitter is a major social media platform commonly used by individuals to spread misinformation. In fact, there have been a mind-blowing 1,641,804,287 tweets regarding COVID-19 from January 22nd, 2020 to February 5th, 2021. In order to control the dissemination of misinformation, the innate systems within Twitter must be further improved to detect and limit the propagation of fake news.

\section{Evaluating the Mechanism of Social Bots on Twitter in the Context of COVID-19}

\section{Toman Aleksiev and Milica Ristovski}

The COVID-19 pandemic has, among other things, brought to light how quickly misinformation spreads. Misinformation 
spreads largely due to social media and the increased amount of time people are spending using social media platforms. However, it is not just people spreading this misinformation, but rather bots as well. Bots, or software robots, are the colloquial term for automated accounts. The particular bots studied in this paper are classified as social bots [1]. Studies have shown that humans have difficulty distinguishing bots from humans [4]. In the past few years, misinformation bots have, like everything else on the internet, evolved and improved. Previously, bots were easy to identify because they posted in regular time intervals and only posted fake news. The goal of this study was to assess the evolution of the mechanism of social bots. Our hypothesis was that bots posted a large quantity of correct, verified, news interspersed with fake news to be more believable and to mimic human behaviour. We also suggested that the frequency of their posts was randomized. Our study analyzed Tweets from Twitter using the Twitter API and was written using Python. The results of this study were inconclusive; however, there is evidence that it should be further investigated. A future direction could include evaluating several bots at once [2] to see if they behave similarly, since bots tend to follow other bots, so such a network may be easier to detect [3].

\section{Infodemiology and Infoveillance of Covid19 using GPT-3}

\section{Robert Joseph}

Fake news detection is the task of detecting forms of news consisting of deliberate disinformation or hoaxes spread via traditional news media (print and broadcast) or online social media. Fake news is especially rampant in the current COVID-19 pandemic, leading to people believing and blindly following in false and potentially harmful claims and stories. Detecting fake news quickly can alleviate the spread of panic, chaos and potential health hazards as well reducing stress and other mental health issues. Using the Generative Pretrained Transformer 3 (GPT-3) which is an autoregressive language model that uses deep learning to produce humanlike text, classify text,design, generate code and various other use cases. Using the classifications endpoint provides the ability to leverage a labeled set of examples without fine- tuning and can be used for any text-to-label task and hence by using various data sets which contain fake and real Covid19 tweets/news GPT-3 was trained on the dataset and achieved a $98 \%$ accuracy by correctly classifying fake news and real news. Apart from using GPT-3 we also used a Passive Aggressive Classifier which is an online machine learning algorithm which also achieved an accuracy of $91 \%$. We also provide future discussions and the limitations of the Deep Learning Model ( GPT-3) as well as the simple Machine Learning model ( Passive Aggressive Classifier). We hope to combat the misinformation of Covid19 spread online with these two models.

\section{An analysis between recreational activities, physical and psychological health for people during COVID-19 outbreaks}

\section{Hang Dong, Jixuan Chen}

We are going to analyze the connection of entertainments, human beings' physical and mental health. In fact, people need to keep social distancing in order to reduce COVID-19 infection rates. And this has to be found to negatively impact people's health by restricting participation in daily activities including travel, outdoor activities or indoor activities such as gyms or parties.

\section{Fighting the COVID-19 Infodemic through Twit- ter Machine Learning}

\section{Ariana Walji, Souraish Mahesh, Irene Ni, and Jes-} sica Yang

As of May 30th 2021, there have been over one- hundred million cases of COVID-19 worldwide [1]. With this growth in cases there was also an increase in the spread of misinformation and fake news, commonly known as the COVID-19 infodemic [1]. To combat this new frontier of the pandemic, this paper examines the bot technology Twitter uses to prevent the spread of misinformation and aims to provide additional perspectives in improving its efficiency and accuracy. We propose improving the current Twitter machinelearning bot through additional search parameters such as hashtags as well as the introduction of phrase-recognition to combat the COVID-19 infodemic. Through a utilization of NLTK processing supported by data processing algorithms using Python and Pandas, data of the most frequently-occurring text features of real and fake twitter datasets are produced, 
which is then put towards a calculation of similarity scores for an accurate categorization of tweets. The patterns in text features that differentiate between real and fake tweets are a key focus in our research and development of the modified Twitter bot. Furthermore, we introduce a novel solution that leverages Twitter bot technology in order for a direct intervention approach that replies to Twitter users who share misinformation with a refuting article from a credible source.

\section{Is it Fact or Fake? COVID-19 Misinformation Tweets and Network Structures are Highly Simi- lar to Factual Tweets}

\section{Adam Radek Martinez, John Zhang, Simha Kalimi- palli, and Karan Manku}

The World Health Organization (WHO) identified false claims and conspiracy theories as a major threat to pandemic control. Therefore, the characterization of COVID-19 misinformation on social media is the first step to minimizing its harmful effects. This study answers the following research questions: (1) How do COVID-19 misinformation tweets influence the structure of discussion networks on Twitter? (2) Are there identifiable characteristics of COVID-19 misinformation tweets? A modularity algorithm for community detection on the CoAID: COVID-19 Healthcare Misinformation Dataset revealed one dominant community containing $15.4 \%-31.3 \%$ of all nodes. Other nodes were distributed among smaller communities making up less than $1 \%$ of nodes. Misinformation was seen less in small communities $(<1 \%)$, and more prominent in the dominant community (6\%). Misinformation tweets generally associate (via retweets and replies) with other misinformation tweets. Principle component analysis and tSNE revealed that misinformation is not correlated to 20 tweet features studied, and highly similar to true information. Trade offs between using larger data sets for machine learning and identifying smaller pockets of misinformation, along with next steps are discussed.

\section{Quantifying and correlating the severity of CO- VID-19 misinformation on Twitter}

Noah Varghese, Rajarshi Mukhopadhyay, David Chen, and Shruthi Sundararaman
Social media platforms are pivotal in public outreach during the coronavirus disease-2019 (COVID-19) pandemic. However, they are also being misused as a means of spreading unverified, unfactual, and even damaging information. Consequently, The World Health Organization (WHO) has labeled this phenomenon an "infodemic". Research surrounding the magnitude of false information circulating on online platforms is evolving; however, the characterization of the severity and gravity of this information is also important. This is because the real-world consequences of misinformation consumed by the public can largely depend on the degree of misinformation independently of the actual magnitude. Thus, our objective was to create a misinformation index (rating) using a coded probability classifier to examine the degree of misinformation regarding COVID-19 within a Twitter data set. The set of pre-labeled tweets ("fake or real") provided by the STEM fellowship was utilized. Misinformed (fake) tweets were assigned an index based on parameters surrounding the author (i.e., verification status, account age, etc). Additionally, this study sought to map correlations between tweet index and specific keywords or hashtags relating to COVID-19 topics. This is done to determine if more or less severely misinformed tweets are correlated to specific topics more so than others. These analyses provide insights into the categorical nature of misinformation that has the potential to cause the most harm. As a result, future efforts by data scientists can be more effectively shuttled towards the management of misinformation that deems the most priority. This approach will more effectively curb the "infodemic" versus fighting all misinformed tweets in an unspecified strategy.

\section{COVID-19 in Canada: A Closer Look at Vaccina- tion Rate using Sentiment Analysis}

Vanessa L., Keyang M., Yuhan X., and Tingyao Z.

The COVID-19 pandemic has had societal impacts in many areas, including news and media transmission. Using the COVID-19 Vaccine Tweets data set, this report explored the correlation between provincial reactions towards vaccines and number of confirmed cases using sentimental analysis and single linear regression. The results showed that there is a linear relationship between the percentage of positive reaction and the vaccination rate (first dose), which aligned 
with the priori hypothesis. The conclusion is that, the more positive Canadians react to vaccinations, the higher the rate of which Canadians receive their first dose of vaccines.

\section{Text Mining and Sentiment Analysis on Real and Fake Tweets of COVID-19 Datasets}

\section{Yang Caiyi}

Based on the COVID-19 healthcare misinformation datasets, I attempt to clean the text for each tweet first by keeping all readable words. Then, by constructing word cloud, it is clear to see the most frequent words existed in thousands of tweets. The pattern that can distinguish between real and fake tweets turns to be more visually comprehensive for us. In addition, I applied sentiment analysis to uncover the emotions behind people who send those tweets. The results demonstrated that negative emotions possess an important aspect in people's mind during pandemic lockdown. It's significant to note that future studies are needed for building a concrete model on identifying whether a tweet is fake or not, under random selection of large numbers of tweets.

\section{CoAID: Detecting Misleading Information Using Deep Learning Models}

Nghi Huynh

COVID-19 virus has rapidly spread around the world and affected lots of peoples' lives. Unfortunately, the diffusion of misinformation related to COVID-19 also gets created and propagates wildly on social media and other platforms. Since the spread of such misleading information has caused many adverse effects on public health, it is crucial to build computerized systems to understand, detect, and mitigate such misinformation. In this paper, we proposed a deep neural network for the detection of fake news. The deep learning models are the modified LSTM with one layer and the modified LSTM with two layers. In particular, we carried out our experiments with a large dataset from tweets and other platforms related to COVID-19. We then separated the dubious claims and news articles into two categories: fake and real. In addition, we set up our baselines with three machine learning models: logistic regression (LR), decision tree (DT), and support vector machines (SVMs). We then validated and compared the performances of these baselines with our LSTM models. The results obtained from our proposed models reveal high accuracy $(89 \%)$ in distinguishing fake tweets from real tweets in the COVID-19 dataset. These results also show a significant improvement in our proposed model as compared to the existing state of art results from the baseline machine learning models. Our findings demonstrate the efficacy and accurateness of the LSTM models in detecting COVID-19 related misinformation and offer a methodological contributions to misinformation detection.

\section{Prevention of Dissemination of False News on Twitter Using Supervised Machine Learning}

\section{Noah Dickinson, Tyler Rivet, Alimot Kolapo, and Helen Balamut}

Rampant spread of misinformation on social media platforms, such as Twitter, has had profound, deleterious impacts on a society that has more readily accessible information at hand than at any point in history. This spread of false information hampers progress towards an advanced public health agenda; including resolution of the ongoing COVID-19 pandemic. This issue must be addressed. To this effect a supervised machine learning model was developed with the intent of distinguishing fake news claims from real ones on Twitter. A prediction model was created by logistic regression that was able to correctly distinguish real news from fake $98.6 \%$ of the time, and with an $80 \%$ accuracy rate based on replies only. These findings have relevance to moderation of social media platforms, given that an increasing number of people turn to them as sources of news. This in turn would help to decrease the level of disinformation permeating modern society.

\section{Predicting Vaccine Uptake Rate Using Machine Learning: An infodemiological Study in the Uni- ted States}

\section{Xingzuo Zhou, Yiang Li1, Shuheng Yang, and Yi- ning Duan}

This paper outlines a framework to monitor COVID vaccination uptake rate. The study uses both statistical and machine learning methods with different classifiers, including LASSO, Support Vector Machine, boost and randomforest, on 
web search queries and clinical data to better predict the vaccine uptake rate in the United States. This paper uses RMSE and scores to compare accuracy, and how the classifier, randomforest, gives the most accurate predictions of the probability that unvaccinated people receive their first dose of COVID vaccine.

\section{Social media's contribution to misinformation in contrast to peer-reviewed articles and the implementation of fact-checking as a counter- measure}

\section{Jeanice Kavena, Kalee Kiluu-Ngila, Camille Lavoie,} and Carlos De la Rosa Martinez

Upon the examination of many social media platforms, including, but not limited to, Facebook, Twitter, Instagram or YouTube, it was found that many viral posts share information that has been manipulated or has not been fact- checked. We found that Facebook was leading with the amount of misinformation spread on this website. Although some news articles and reports also contribute to the spread of misinformation, they were found to be much more factual in nature than social media. With much of the public relying on social media and on other web pages, the increased manipulated data they encounter can have a dangerous effect on mental health, as well as increasing sen- timents of fear and racism. Evidence of the detrimental effects can be observed in several social movements that were created to counter the safety precautions that are being endorsed and enforced by the government.These countermovements include Anti-maskers, Anti-vaxxers, and groups who participate in protests against lockdowns. To counter the spread of misinformation, we suggest that the social media websites listed hire workers to attach fact-checked reliable sources to the viral posts. We also suggest readers read these articles (or find some of their own from reliable sources if none are yet attached to the post) before making any decisions regarding their physical health. These simple solutions would allow social media users to stay on top of the misinformation being spread. We suggest that future research should be done on building social media algorithms to automatically flag possibly misleading posts.

\section{Using Machine Learning Methods To Predict Fake News \& Exploring Vaccine Manufacturer Sentiment}

\section{Raymond Romaniuk}

With 2.5 quintillion bytes of data created each day [1], the spread of misinformation is more relevant than ever. Big data requires big responsibility and we must do our best to slow the spread of false claims that may turn to public health risks. Our paper aims to use machine learning methods to accurately classify fake news headlines to stop the spread of potentially dangerous health narratives. In addition to this we explore the sentiment shown towards COVID-19 vaccine manufacturers Pfizer, Moderna and AstraZeneca. We use two datasets for our analysis. The first containing news headlines shared on social media and labelled as real and fake news. The second containing vaccine related tweets. We use machine learning methods, like logistic regression and support vector machines, to attempt to classify news headlines as real or fake news. Additionally, we explore the uses of clustering algorithms and neural networks. To determine the sentiment of our vaccine related tweets we use Python's Natural Language Toolkit. With our estimated sentiments we fit time series models to forecast future public sentiment. We found that the Kernel Support Vector Machine was most accurately able to predict whether the news headline provided was real or fake news. It's accuracy was substantially higher than any of our other models at $73.46 \%$. We also observed that our sentiment analysis of vaccine related tweets successfully identified the impact of negative news stories on a manufacturers public sentiment. For example, the reports that the AstraZeneca vaccine may cause blood clots [2] visibly increased AstraZeneca's negative sentiment. The fake news classifier we explored may be a helpful addition to large social media platforms like Facebook, Twitter and Instagram. The development of an accurate model could limit the spread of potentially dangerous false health claims and create a safer social media environment for all.

\section{Combating Misinformation: Solving Twitter's COVID-19 Infodemic Crisis using Computational Methods on Open Source Databases}




\section{Alena Ho, David Woo, Siddharthan Lakshmanan,} and Silvanus Kolade

During the COVID-19 epidemic, a proliferation of misinformation on Twitter has led to unregulated false tweets. This has negatively impacted the public and has been deemed an 'Infodemic' by the World Health Organization (WHO). Using open source databases, tweets with true and false information were coded in Python and the most frequent terms were analyzed. False tweets were found to have more sensational and violent terms whereas true tweets were more inclined to be work and travel related. This suggests that false tweets purposely promote misinformation for public distress whereas true tweets focus more on adapting to quarantine restrictions. A suggested solution for regulating false tweets is a flagging system. This match system would deem tweets false by how often the terms used were found in a database of false tweets. If multiple tweets from the user is flagged, the user would also be penalized. This solution can be further expanded on by in investigating the public's reaction to being regular exposed to proliferated of misinformation. The findings and solutions in this study could also help prevent simi- lar instances in future epidemics by encouraging greater regulation on social media platforms.

\section{Positive or Negative? A Sentiment Comparison Between Real and Fake COVID-19 News Articles}

\section{Justin Heer, Ryan Kelly, and Leo Chen}

COVID-19 pandemic has created a large amount of misinformation and misinterpretation of scientific facts. Our paper analyses and compares the sentiment of real and fake news articles, allowing classification of articles as either real or fake based on the sentiment portrayed in the text. We perform web scraping using labelled news articles hosted in the CoAID dataset to collect article content. We utilize SUMY, a python package for article summarization to extract key components of articles for analysis. Then we process the summaries using vaderSentiment, a lexicon and rulebased sentiment analysis tool [1] that provides estimates on the articles sentiment. The sentiment analysis indicates that the real articles have positive sentiment (compound score $=0.13464)$ and fake articles have negative sentiment (compound score $=-0.05618$ ); the difference is statistically significant with a $p$ value of $6.05 e-22$.

\section{Predicting the Emotional Valence of COVID-19 Related Tweets}

\section{Wilson Tu, Shuaib Habib, Alexander Adrian-Ha-} mazaki, Tarang Mahapatra

During the COVID-19 pandemic, social media has been a cata- lyst in the dissemination of information. This study investigated distribution patterns of news utilizing data from Twitter. Tweets with key words and phrases related to the pandemic were identified, and characterized based on their emotional valence using a machine learning algorithm. Additionally, natural language processing was utilized in order to examine the usage of different words to further characterize the sentiment of a tweet. Using these characterizations, patterns of their dissemination were analyzed. The analysis demonstrated a slight correlation between the sentiment of a 'trending' tweet and its' popularity, however, this does not hold when all tweets are considered. Utilizing our findings, we propose a more precise and refined machine learning model1 . Targeted investigations into patterns of dissemination may aid in stopping the spread of misinformation, thus supporting the public health measures for the COVID-19 pandemic.

\section{Machine learning-based predictive modelling of COVID-19 vaccination uptake within U.S. coun- ties}

\section{Queena Cheong, Stephanie Quon, Katsy Concepcion, and Martin Au-Yeung}

While the COVID-19 pandemic has left an unprecedented impact globally, countries such as the United States of America have reported the most significant incidence of COVID-19 cases worldwide. Within the United States of America, various sociodemographic factors have played a vital role in developing regional disparities. The presence of regional disparities has resulted in the unequal spread of disease between US counties, underscoring the need for efficient and accurate predictive modelling strategies to inform public health officials and reduce the burden on healthcare systems. Furthermore, despite the widespread accessibility of COVID-19 vaccines across the United States of America, 
vaccination rates have become stagnant, necessitating predictive modelling to identify factors impacting vaccination uptake. This study utilizes sociodemographic data and machine learning to develop a predictive model for COVID-19 vaccination uptake on a county level. Sociodemographic data on fully vaccinated and unvaccinated individuals were sourced from several online databases, such as the United States Centre for Disease Control and United States Census Bureau COVID-19 Site. Machine learning analysis was performed using XGBoost and sociodemographic data. Our model predicted COVID-19 vaccination uptake across US countries with 59\% accuracy. The model also identified important sociodemographic features used to predict vaccination uptake; location, education, ethnicity, and income. Lastly, the model produced a visually accurate choropleth- which can be used by authorities in future pandemics to visualize and prioritize areas of low vaccination and design targeted vaccination campaigns.

\section{Data Exploration and Classification of News Article Reliability: A Deep Learning Study}

\section{Kevin Zhan, Rafay Osmani, Yutong Li, and Xiaoyu Wang}

During the COVID-19 pandemic, huge amounts of new information from many different sources is made available to us each day. This pandemic has been worsened by this associated "infodemic". As individuals are becoming increasingly reliant on the internet as a source of information, the presence of unreliable information or misinformation can become a source of confusion. This ultimately leads to misunderstandings of public health orders or even indirectly affects public policies. While it is critical to identify misinformation and unreliable news sources online, this task becomes increasingly challenging with the tremendous amount of information released each day. We propose the usage of natural language processing (NLP) and machine learning (ML) techniques to build a model that can be used to identify unreliable articles online. We first processed the ReCOVery dataset to obtain 2039 English news articles tagged with COVID-19 keywords from January to May 2020 which are labelled as reliable or unreliable. Data exploration was done to determine major differences between reliable and unreliable articles. We found that reliable news articles had a higher proportion of neutral sentiment while unreliable articles had a higher proportion of positive sentiment. Additionally, our analysis demonstrated that reliable articles had more engagement on social media in terms of tweets, retweets and likes. We built a multi-input deep learning model called Deep- Reli using sentiment and body text of articles to classify the reliability. DeepReli was evaluated to achieve a $82 \%$ accuracy, $82 \%$ sensitivity, $81 \%$ specificity, and 0.84 AUC score. This paper identifies novel differences between reliable and unreliable articles as well as builds a model using state-of-the-art deep learning techniques. We aim to be able to use our findings to help researchers and the public audience more easily identify misinformation and unreliable me- dia in their everyday lives. Future work by researchers could include creating a larger multi- input model which includes features such as geographical location as well as time-series data of news articles. Additionally, it would be valuable to compare the efficacy of the model on other media such as social media posts or headline as well as with different news articles such as those discussing major political events.

\section{Investigating the Effect of Highly Discussed To- pics in TWITTER on COVID-19 Vaccination}

\section{Leyi Wang, Xiaozhou Ye, Zhuoran Li, and Jiayi Zhu}

Although COVID-19 is not the deadliest virus the human race has ever encountered with a case fatality rate of approximately $5 \%(J o h n s$ Hopins 2021), the pandemic affected a tremendous portion of the entire population. Fortunately, with the advancement in pharmaceutical fields of study, scientists were able to synthesize vaccines that help train our human bodies to gain immune response to the virus. While developing successful vaccines are important, delivering the information to the public correctly and timely is also very crucial. It is believed that Twitter is faster than the News in terms of spreading (both true and false) information (Leetaru, K. 2019), and here we are interested in the effect Twitter has on the COVID vaccination. To analyze, we applied simple linear regression and generalized additive model (GAM), which is a combination of generalized linear model and additive model, on relevant data. Through data analysis, we observed 
a relatively positive cor- relation between the number of COVID-related hashtags and the rate of vaccination, and a linear relationship between positive sentiment of the tweets and the rate of vaccination. We came to the conclusion that properly making use of the features of Twitter (hashtag, re-tweet, etc.) may serve to increase awareness of the information in the public.

Investigating the impact of claims on AstraZeneca vaccine causing blood-clotting on the public perception of vaccination

\section{Jeyoung Oh, Trista Tian, Thao Tran, Max Vu}

The claim that AstraZeneca vaccine may come with the risk of blood-clotting have had an impact on the public perception of vaccination. In the period where there's an infodemic that divides public opinion, vaccine hesitancy continues to persist due to fear and negativity evoked by such claims. Through this study, the magnitude of public reaction to negative news about the AstraZeneca vaccine is investigated through examining activity data on social media platforms such as Twitter and YouTube. Using wordassociation analysis, sentiment analysis and statistical analysis, the shifting perception of AstraZeneca vaccine and other brands of vaccines are quantified and correlated with news event. Negative news on AstraZeneca evoke strong negative receptions from the public, while positive news did not evoke much positive receptions at all. The investigation demonstrated that the public saw the claim around AstraZeneca association to blood-clotting risk a significant piece of information that also influenced their opinions on vaccines and vaccination in general.

\section{Evaluation of Twitter's Preventative Measures on the Spread of Misinformation on the CO- VID-19 Vaccine}

\section{Sina Haghighi, Glenn Chang, Kamakshi Naidu and Harleen Kaur1}

Twitter has increasingly been the hub for people to obtain daily news and information, yet it is plagued with misinformation and fake news. During the coronavirus pandemic, information regarding the COVID-19 virus and health policies were constantly changing and it was difficult to differentiate between real or fake news. As such, this poses a serious health risk to society. Twitter has taken steps to combat this by utilizing machine learning to detect and delete misinformation, adding labels to misleading tweets and adding an exploration tab for accurate COVID-19 information. Our study aimed to investigate the effectiveness of these policies by looking at the frequency of COVID-19 vaccine misinformation before and after Twitter added these policies. As a proxy measure to quantify misinformation, we examined the tweet sentiment and subjectivity value, and found trends in common words and hashtags. In this preliminary study, we have found a general decreasing trend in misinformation after Twitter implemented these preventative measures suggesting that these policies are effective. Further study will need to confirm this as major assumptions in misinformation and sentiment value were made and various confounding variables may have affected the data. By studying Twitter's misinformation policy during COVID-19, we hope other social media companies will continue to combat misinformation.

\section{What pandemic events have had the most influence on public sentiment towards the Ast- raZeneca, Pfizer and Moderna COVID-19 Vacci- nes?}

\section{Muskaan Kaur Bajwa, Angelica Ramoutar and Neha Purakan}

Vaccination of the general public is a key step towards fighting the spread of COVID-19. Since the start of the coronavirus pandemic in March of 2020, there have been numerous events that have influenced the nations' attitudes towards Pfizer, Moderna, and AstraZeneca vaccines. News relating to vaccine efficacy, vaccine distribution, vaccine clinical news, alternative drug news, government guidelines, and coronavirus transmission news impacts the public's opinions on the vaccines. As such, the ability to investigate changes in the public sentiment towards vaccines in response to news events can act as a powerful tool that can be utilized to understand the underlying causes of an infodemic, and it can also provide some further insight into public behaviour. To identify and investigate which events throughout the pandemic have affected people's sentiments towards the Pfizer, Moderna, and AstraZeneca vaccines the most, a Twitter sentiment analysis as well as a stock price analysis 
was performed. A data set of 4,093,082 tweets containing the key phrases or hashtags related to the AstraZeneca, Pfizer and Moderna vaccines were compiled from February 25, 2020 , to May 25, 2021, using the Twint python tool. Sentiment analysis was conducted using the Valence Aware Dictionary and Sentiment Reasoner (VADER) tool[1]. The Stock Price data collection was extracted from Yahoo Finance over the period starting from January 29, 2020, to May 28, 2021. Timelines of influencing events were constructed by identifying days for which a significant change in stock price or tweet sentiments occurred and then matching those days to significant events related to the pandemic. Analysis of tweets revealed that events that are associated with significant changes in the positive, negative, and neutral sentiments towards the three vaccines are the imposition of government guidelines related to quarantining and travel, events relating to vaccine company business relations and the increasing transmission of COVID-19 respectively. Our findings suggest that to combat the spread of misinformation, more clarity regarding how vaccine companies operate is necessary.

\section{A Sentiment Analysis of Public's Attitude on Social Media Towards the COVID-19 Vaccines}

\section{Brandon Van, Gwyneth Yeung and Megan Ng}

COVID-19 has been classified as a global pandemic by WHO on the 11th of March 2020. Since then an immense amount of funding and research has been poured into finding a vaccine for COVID-19 leading to many different vaccines being released to the public. Some of these vaccines are more conventional however some such as the Pfizer vaccine utilize mRNA instead of the traditional protein conjugates. The fast release of these vaccines have led to some concerns from the public. Many of these concerns stem from the possibility of unknown long term side effects, but some concerns also come from the anti-vaccination movement. This spread of misinformation during a pandemic has led to the coining of the term infodemic which is defined as the rapid spread of information that can weaken the response to an outbreak. In the information age there is an enormous amount of information available and very few ways to effectively moderate the information. This is dangerous as it leads to many believing erroneous facts that can cause irrational actions. One example of this is Donald Trump claiming that injecting cleaning agents such as bleach would help defend against COVID-19. Many past studies have used social media to investigate attitudes of the public towards various government initiatives that are aimed at stopping the spread of COVID-19, however due to the novel nature of the vaccines there are not many studies looking at the attitudes towards the vaccines. The objective of this paper is to investigate public attitude towards the COVID-19 vaccines looking at Twitter. A fine grained sentiment analysis was conducted using NRC Sentiment and Emotion lexicon on a data set containing tweets filtered by hashtag CovidVaccine published from 01/082020-04/21/2021.The tweets from the data set were compared with a list of manually annotated words associated with 8 emotions and 2 sentiments, scores for each emotion and sentiment were then computed to understand the attitudes of the tweets. The data demonstrated that most reacted positively to the vaccines with the most notable emotions being anticipation, joy, and trust. Based on this data, it can be inferred that most people are accepting of the COVID-19 vaccines, however to expand the study to more accurately encompass everyone other social media such as Facebook and Instagram should be included in the future. A holistic view on the public sentiment will aid authorities in identifying ways to help the public gain acceptance in the COVID-19 vaccine hence achieve herd immunity.

\section{Impact of social media platforms on sale trends in COVID-19}

\section{Kashish Aggarwal and Vansh Joshi}

The following research paper explores the tweets from the popular microblogging social networking site Twitter and analyses them into finding a pattern in sales of products during the different stages of COVID-19 pandemic. The study then further goes on to establish how the infodemic and dearth of misinformation available online is just as harmful as the ongoing pandemic because this infodemic builds on the fears and ignorance of the public and leads them into panic buying. The need of the hour is for the social media platforms to be vigilant in their surveillance and supervision of data so that they can prevent misinformation from spreading. Similarly the consumer today is expected to be informed and not contribute to the pool of false information available online in a health crisis like COVID-19, while also 
preventing themselves from falling trap to mass mentality of panic buying.

\section{The Correlation Between News Media and Web Searches with COVID-19 Sentiments on Twitter}

\section{Alvin Hendricks and Rayyan Zuberi}

Over the past year, COVID-19 has left the public emotionally polarised. An open access dataset containing Sentiment Analysis of more than $1,637,526,681$ Twitter posts related to COVID-19 was used to determine daily average Sentiment. The time period considered spanned from January 22, 2020 to May 2, 2021. Interest in key search terms were also surveyed through Google Trends. It was determined that average Sentiment was consistently negative throughout the pandemic, but had a generally upward trend. Spikes in average Sentiment were observed to be correlated with pivotal American and international news events. Spikes were additionally correlated with increases in the relative interest in certain search terms over time. Results suggest that relative interest in key terms and news articles may be a predictor of Sentiment. This relationship corroborated with level of policy compliance could be used to predict and improve implementation of future public health strategies. Areas for further research include expanding analyses to other additional social networks to reduce potential biases.

\section{Classifying Fake COVID-19 Tweets With Supervi- sed Learning and Deep Learning Models}

\section{Mashiyat Saif, Marcus Chung, Oluwatitomi Adebajo and Oluwatobi Adebajo}

Fake news is prevalent online due to the numerous social networks offered on the internet. The COVID-19 pandemic started a new wave of health-related fake news that has increased the spread of misinformation. The tweets obtained from the "CoAID": COVID-19 Healthcare Misinformation Dataset contains healthcare information related to COVID-19, their reliable labels, and user engagement features. This paper applied machine learning (ML), deep learning (DL) and transformer based ML models (BERT) on the dataset. The dataset was first extracted using the computer software Hydrator, and included 252471 tweets from December 2019 to September 2020. The data was then preprocessed and vectorized. Exploratory analysis of the raw dataset was first done in Excel to find the correlation between the different tweet features. Classifying the tweets as "real" or "fake" was done in three ways: via Traditional Supervised Learning models, Traditional Natural Language Processing (NLP) models, and Deep Learning models. Traditional Supervised Learning models involved selecting variables about the tweets such as number of retweets, and using those features for classification. The next method was to apply traditional models such as Naive Bayes (NB) and Random Forest (RF) models to the vectorized data. Finally, two Deep Learning models were created: a Deep Neural Network (DNN) model and a Bidirectional Encoder Representations from Transformers (BERT) model. Overall, the SVM and RF NLP models provided the best results on the test data. The two DL models also performed similarly and effectively classified fake news from real news, although with slightly less accuracy and recall. The findings from our analysis demonstrates that various models are available to suitably classify information. An example of the deployed model our team created called "TweetScan" can be found here. It ultimately illustrates how the results of this paper can be applied to reduce the spread of misinformation, especially concerning recent issues such as vaccination acceptance.

\section{Investigating the role of Misinformation on Twitter through Passive-Aggressive Classifica- tion}

\section{Scott Blender, Kelly Ly, Hadassah Galapo, and En Yu Yap}

In this paper, we consider the problem of misinformation spreading on Twitter. We collected a large dataset of more than 1.2 million tweets related to the topic of the COVID-19 vaccine from May 3rd, 2021 to May 24th, 2021. We trained a binary classification model to detect whether a tweet was considered misinformation or not misinformation. The Passive-Aggressive Classifier (PAC) outperformed logistic regression and was selected to classify the entire dataset. Overall, $79.83 \%$ of tweets were classified as not misinformation and $20.17 \%$ as misinformation. Our results also highlight the distribution of sentiment and the most popular terms in both the tweets classified as misinformation 
and not misinformation. Through our study, we propose a workflow to assess COVID-19-related Twitter behavior using misinformation classification, topic analysis, sentiment analysis, and retweeting behavior. From our findings, we suggest that platforms like Twitter establish policies to reduce the spread of misinformation and that public health officials inform the public of key topics that appear frequently in misinformation tweets.

\section{Comparing the Differences in COVID-19 Topic Discussions in Science, Media, and Public}

\section{Kate Kim and Myriam Lizotte}

The COVID-19 pandemic is an opportunity to study infodemiology, i.e. the spread of public health information. We can leverage large data sets on COVID-19 to investigate how the general public accesses these scientific findings. We hypothesize that scientific information, especially relating to public health, flows from the scientific literature to the general public via the media. To test this hypothesis, we aim to analyze the discourse on COVID-19 in three different areas of communications: (1) the scientific community, measured by publications in scientific journals, (2) the media, measured by news articles, and (3) the general public, measured by tweets from Twitter. In particular, we select four prominent topics relating to the COVID-19 pandemic (face masks, vaccines, the origin of the virus, and public health restrictions) and evaluate their prominence in each three areas. However, our comparisons did not support our hypothesis. The most common topics in the scientific literature were independent from those in news articles. Reducing these differences in discourse may be the first step in improving access to quality scientific communication.

\section{Epistemic Bubbles: A Network Analysis of the Social Conformity Presented by the COVID-19 Infodemic}

Ethan Rajkumar, Sandra Radic, Jubelle Paa, and Terry Lin

Misinformation spreads fast, quite possibly faster than disease - especially with the powerful tool of social media. During the COVID-19 infodemic, individuals have experienced this rapid spread of misinformation and suffered from its' consequences. Misinformation tends to circulate through communities in the form of epistemic bubbles, or in other words, conspiracy-confirming resources. People who belong to epistemic bubbles are surrounded by sources that validate each other, causing communities to sometimes misplace their trust in fake sources. Unfortunately, little to no publications have discussed the phenomena of epistemic bubbles at a time where COVID-19 misinformation exponentially spread on social media, especially on Twitter. To address this knowledge gap an extensive analysis of COVID-19 related Twitter misinformation data was conducted. Geolocations obtained from 6273 tweets relating to COVID-19, were placed into a world map spanning from May 1st to November 1st 2020. Using a repository of tweets and news articles provided, the amount of tweets that shared a specific URL were studied using network analysis and community visualization. It was concluded that there were epistemic bubbles that could be arranged into tweet levels depending on the number of users that shared common fake news articles within the epistemic bubbles. Next, a general trend towards highly vascularized pandemic epistemic bubbles, was shown during the period from May 1st to November 1st, 2020. Based on these findings, this study can suggest promising directions for future investigations. Through the results of this analysis and after further optimization, governmental policies can be put in place to expose misinformed users to different credible resources, breaking these epistemic bubbles.

\section{Fighting the infodemic: Leveraging Supervised and Semi-Supervised Machine Learning Model to detect Fake Tweets on COVID-19 pandemic}

\section{Sabiha Sultana, Prateeksha Aggarwal, Ruini Xiong, and Rucha Acharya}

The spread of misinformation is highly prevalent in the present day, arising from the current infodemic. Large sources of these fake news are various social media platforms. Twitter is one of the biggest social media platforms in North America and is a hub for the spread of information. Distinguishing between fake and real data on Twitter can be difficult due to the lack of labeled data as the process to do this is time consuming and costly. Using data sets containing 10700 twitter status updates, a combination of supervised and semisupervised models were created in order to make the act of 
labelling data more accessible and to create a model that is more accurate than just a semi-supervised model alone. Labelled data from the data sets was put through logistic regression to create the supervised model. Following this, a semi-supervised model was generated using label spreading where the previously labelled data was combined with unlabelled data. This combined data was once again analyzed using the logistic regression model in order to determine how accurately the unlabelled data was classified. The accuracy of the first logistic regression supervised model was the highest at $96.5 \%$. Label spreading alone had an accuracy of $90.7 \%$, which was the lowest out of all of the models. Combining label spreading and logistic regression gave an accuracy of $92.9 \%$, which was between the accuracies of running only a supervised model and running only a semi-supervised model. Based on these results, this study concludes that using a combination of the supervised and semi-supervised models produces a higher accuracy than solely using a semisupervised model. Through this combined model, fake and real data can be categorized and labelled, to prevent the spread of misinformation during this infodemic.

\section{The Method to analyze the trend of the fake and fraud news of COVID-19}

\section{Xingyou Zhou, Yupeng Zhang, Weilin Fu, and Jing- jia Meng}

COVID-19 has interfered with people's daily lives for a long time. During this time, everyone is stressful which causes them to have some unrealistic thoughts. This paper outlines a framework to analyze the behaviours of the "Netizens" and the media. The analysis illustrates the significance of the media on social media that how they lead the ideas of "Netizens" and predict the future behaviours of "Netizens".

\section{Classifying sentiments towards COVID-19 vacci- nes through supervised learning}

\section{Anna Jeffs, Wendy Ma Zhang, and Kisa Naqvi}

The onset of COVID-19 has resulted in the rapid development of potential vaccines. However, public opinion towards these new vaccines has been varied. These opinions are commonly expressed on Twitter, which is why this report fo- cuses on identifying and classifying them by sentiment. This report establishes a dataset of $2000+$ tweets regarding COVID-19 vaccines and uses them to train and test a logistic regression model that can be used to classify tweets as positive or negative. This can then in turn be used as a tool to gauge public opinion as vaccine development and distribution progresses. While models to identify sentiments in tweets already exist, this is the first to focus solely on COVID-19 vaccine opinions. As a result of this, it is better suited to some of the vocabulary specific to vaccines. The majority of tweets regarding the COVID- 19 vaccine are neutral or positive in nature. Negative tweets account for less than $20 \%$ of the collected sample. While they are not as prevalent, negative tweets still have the potential to discourage those from taking the vaccine which could in turn negatively affect the overall health of society. This model could also be used to identify and contain material that could potentially damage peoples' perception of vaccines. Though this research is preliminary, the results are promising for the analysis and monitoring of public opinion towards COVID-19 vaccines. Further study should be conducted to expand upon these findings to improve the accuracy of the model and potentially help better COVID-19 vaccine awareness.

\section{An Analysis of the Relationship Between the Online Spread of COVID-19 Vaccine Misinforma- tion and Public Sentiment during the COVID-19 Pandemic}

\section{Nicholas Chronis, Charles Chu, Yunbo Jiang, and Olamide Olanrewaju}

The spread of misinformation during the COVID-19 pandemic has affected the way in which people interpret and react to publicly available information about the COVID-19 virus and vaccination. Social media is an outlet where millions of people interact daily about news, interests, and their social lives. These platforms are undoubtedly beneficial as they connect people across the globe and facilitate dissemination of information. However, it can be extremely challenging to differentiate facts substantiated by scientific data from erroneous information being circulated. The current pandemic has led to government-imposed lockdown and quarantine measures to help curb the spread of the virus. Unfortunately, government restrictions have led to greater reliance by the 
public on social networks for their primary source of news, which has created a glaring issue of an infodemic whereby the public is inundated with vast amounts of information, oftentimes confusing and conflicting. This has contributed to diverse and opposing perspectives about vaccination. Our study aims to investigate the relationship between public exposure to false information about the COVID-19 vaccines and its effects on public sentiments regarding vaccines. False information was differentiated from factual information via posts on Twitter through machine learning and was compared to public sentiments determined using VADER analysis. The results of this study could aid in determining the effects of misinformation on the public's trust of health authorities, which could inform decisions to regulate the availability of misinformation on online platforms. Future directions of our study could include analyses of possible secondary relationships between public sentiments and vaccination rates.

\section{A Hybrid Deep Learning Architecture to Detect Fake COVID-19 News on Social Media}

\section{Jia Yi Ong}

False or inaccurate information that circulates social media platforms can instill false beliefs and encourage illinformed decisions. This can have adverse effects on the society as a whole, especially during the pandemic period. Thus, it is imperative to utilize the latest techniques in Artificial Intelligence and Analytics to curb the spread of misinformation. I employ a sequential deep learning network with Word Embeddings-based feature extraction to detect false and inaccurate social media content such as Facebook posts and Tweets. My hybrid architecture leverages the strength of Convolutional Neural Nets (CNNs) at feature learning and the insensitivity to gap length of Long ShortTerm Memory (LSTM) models. I train my model on a COVID-19 social media data set to learn the semantic structures within various lengths of Tweets or other texts on social media for high-performance detection of fake news. My tuned model yields a test accuracy of $86.88 \%$ and a True Positive rate of $88.76 \%$. Further refinement to the hybrid architecture and parameter tuning should yield higher performance closer to that required of a real-time fake news detector.
How political ideologies influence the engagement in COVID-19 misinformation of Twitter users

Maggie Ye, Srinidhi Shankar, Ginny Guo, and Sagarika Rabindranath

The COVID-19 infodemic is an ongoing challenge perpetuated by social media, with Twitter being one of the platforms that is contributing to the spread of misinformation. A survey done by the database company, Statista, shows that the majority of Twitter users are within America [1] and Americans are also "super spreaders"[2] of COVID-19 misinformation. In this paper, we examine the effects of a user's political ideology on their participation in the spread of misinformation. Based on previous research $[3,4]$, we expected that Twitter users with a conservative political ideology would be more susceptible to spreading misinformation as opposed to their liberal peers. Initially, tweets containing information about COVID-19 that included the keywords "Biden" or "Trump" were collected. These were sorted by whether or not the information in the tweet was accurate. We were then able to estimate the tweet's political ideology by calculating the post's sentiment score. Finally, we used favourite counts for the tweets to determine the relationship between users' political allegiances and their engagement with misinformation. Our results suggest that there is no definite correlation between fake claims on Twitter and favourited tweets, regardless of the user's political ideology. However, to further explore user engagement in these tweets, the sentiments of their replies can be analyzed.

\section{The Effect of the Spread of Anti Vaccine Ideolo- gy on the Internet in the Rise of COVID-19 Cases}

Aditi Das, Tanya Ghai, Mit Patel, and Rudra Patel

Coronavirus disease or COVID-19 has been a catalyst to redefine the way of life around the world. It was classified as a world-wide Public Health Emergency by the World Health Organization (WHO) on 30 January 2020 (CPHA, 2021a). Ever since, there have been many governments as well as pharmaceutical companies that have tried to find a vaccine to help tackle the exponentially increasing number of cases of COVID-19 around the world. Among those there 
are the mRNA vaccines like Pfizer, Moderna and viral vector vaccines (Centers for Disease Control, 2021). There has been extensive research put into determining the effectiveness of these vaccines. However, vaccines have been a controversial topic on the internet and more precisely in various social media platforms. There is a growing community of those who classified themselves as anti-vaxxer who are hesitant about taking the vaccines for various reasons in- cluding their skepticism about the effectiveness, fear of fatal side effects or firm belief in many conspiracy theories. This paper analyzes the use of hashtags and search terms on social media that contributes to the spread of COVID-19 misinformation and uses Python and Excel as well as external sources to gather graphical representations of the data. These trends are then studied to determine the effects of the rate of spread of false information on the rise of COVID-19 cases. It was found that on twitter there is a higher rate of occurrence of hashtag covid vaccine, however any sort of anti-vaccine hash- tags are blocked by the social media platform, which prevents the spread of misinformation to an extent. Sentiment analysis of about 207006 tweets showed that while the majority of users had a positive attitude towards the COVID vaccine, there were a significant number of people who were skeptical about the vaccine. Finally, analysing google trends show a surge in the use of search terms related to anti-vaccine ideology in December 2020, right after the COVID vaccines were first approved by FDA. This again confirms the presence of public mistrust towards the vaccine. There are some limitations to our study, enough data such as the location of false tweets needs to be collected to prove the correlation between the spread of misinformation of anti-vaccine ideology and the rise of covid cases in certain locations, finding the number of bot accounts on Twitter which is contributing to spread of misinformation, and access to online resources, databases, surveys, and questionnaire. The study performed in this paper would be an informative source of information for related studies in future and implement ways to reduce the spread of misinformation.

\section{Visualizing Regional COVID-19 Vulnerability Ba- sed on Misinformation Prevalence}

Sina Allen, Jessica Son, Tony Sun, and Matheus Zhu
The overflow of correct and incorrect information has been affecting the citizen's lives more than ever after the pandemic hit them. As Abraham Lincoln said, "Let the people know the facts, and the country will be safe", the accuracy of the information being spread to the public takes high importance in the citizen's wellness and safety. To validate this assertion, this paper attempted to visualize the regional COVID-19 vulnerability with correlation to the regional misinformation prevalence. Using the datasets from various online sources and projects, the Fake News Per Capita and Cases Per Capita were calculated to represent the regional COVID-19 vulnerability. The prevalence of misinformation was calculated to compare with the Cases Per Capita values. As a result, the linear regression showed a positive correlation between the two values, showing an accuracy of $12.87 \%$. Considering various factors that contribute to a region's infection rate, its partial prediction could be made using the predominance level of misinformation.

\section{Examining the Impact of Social Media on the Choice of COVID-19 Vaccine in the USA - A Senti- ment Analysis Based Study}

\section{Seokhyoun Hong, Yejin Park, Rasesh Dawonauth,} and Siyul Byun

The vaccines against the COVID-19 pandemic have been actively rolling out in various countries around the world, and many vaccine companies are releasing their products. At the same time, countless pieces of information about the disease are being shared on social media even though their credibility has been questioned. Thus, the WHO has announced a COVID-19 infodemic, an overabundance of information [1] that may influence the decision making process of the types of vaccines received by individuals. We studied the relationship between the Twitter sentiment analysis data and the number of vaccine doses taken in the USA from each specific vaccine company. The Twitter data was distilled by the factors of location and the inclusion of the names of the vaccine companies within the tweet's text. A human language processing algorithm was applied to the tweets to get the polarity score representing the overall sentiment of the text. The obtained sentiment values of individual tweets were then adjusted by the follower count of the writer. The resultant weekly trends of Twitter sentiment on vaccines 
were compared with the weekly vaccination counts in the USA via correlation tests. The correlation tests performed for the vaccine companies of Moderna, Pfizer, and Johnson and Johnson showed the respective correlation coefficients of $-0.2457,-0.1078$, and 0.5631 ; thus, not allowing us to reject the null hypothesis. The study was unable to derive significant evidence that social media affects people's vaccine choices. Further studies are advised to take into account various possible confounding variables such as socioeconomic status, age, gender, and cultural background.

\section{Fear of COVID-19 Vaccine Hesitancy using Senti- ment Analysis and World Population Data}

\section{Oluwatimilehin Ajayi, Gurmol Sohi, and Joshua George}

Vaccines are touted to be the most successful public health measure and they have been around since the $1800 \mathrm{~s}$ and vaccine hesitancy likewise. COVID-19 (severe acute respiratory syndrome coronavirus 2 (SARS-CoV-2)) is a viral infection that likely originated in bats and was transmitted to humans, this disease first appeared in Wuhan, China and has spread across the world resulting in the world health organization declaring the virus a pandemic. With many governments, and organizations invested in returning to life pre-COVID-19 (without masks, governmental restrictions, lockdowns etc.), vaccines were developed namely the Moderna, Pfizer-BioNTech (Pfizer), Oxford-AstraZeneca (AstraZeneca) and Johnson Johnson vaccines. Just like the groups who are invested in slowing the spread of COVID-19, there are other groups who look to benefit from COVID-19 by spreading information and misinformation about the virus, its pathology and the new vaccines. Through infodemiology, this report aims to address myths and provide answers relating to the COVID-19 vaccines. Data was collected from research papers, websites, and kaggle generated twitter datasets. They were then analyzed using Jupyter, and Matlab, Numoy, Textblob, Scipy, Pandas and Seaborn to determine polarity and subjectivity of the datasets.themes and extract patterns of knowledge reflecting ideologies behind the beliefs of those content creators. Although the extraction of twitter datatweets had a neutral polarity and marginally positive subjectivity showed a neutral data set, there however was however a strong positive relationship between vaccine fear and vaccine hesitancy. The neutrality of the twitter dataset may conceal the polarity of beliefs both extremely for and extremely against the vaccine. Fear of vaccinations is proving to be a huge public health concern because it signals that the public has low confidence in science and modern medicine leading to a growing population that is vaccine hesitant. We provide a possible reason a growing number of the population is fearful about vaccines, misinformation (infodemic), and conclude that scientists, corporations and governments must do more to eliminate fear of vaccines and that will in turn decrease the amount of people who are vaccine hesitant. We recommend future studies to take stock of different cultural, socioeconomic, and religious populations and their attitudes towards vaccinations and take steps to restore the confidence in the safety, efficacy and utility of vaccines against diseases and viruses like COVID-19.

\section{Covid-19 Fake News Detection}

Yuyan Chen, Zijun Zhao, Dayou Ren, and Xichen $\mathrm{He}$

Fake news detection is crucial for addressing misinformation on social media. This project aims to propose machine learning models for fake news detection based on text mining. Three models, naive Bayes, logistic regression, and random forest, are trained based on term-frequencyinverse document frequency (TF-IDF). The effectiveness of text mining- based models is justified with model accuracy. Possible improvements for future studies are suggested based on the discussion of limitations of text-mining based models.

\section{You've Reddit All: Popular COVID-19 Topics and Public Sentiment Trends in Vancouver, British Columbia}

\section{Cathy Yan, Melanie Law, Stephanie Nguyen, and} Janelle G. Cheung

Social media has become an inseparable part of how we rapidly consume and digest health related news associated to COVID-19. Popular social media platforms have become a ground for heated debates and discussion surrounding topics of importance to users of these sites. To determine its effects on public sentiment, natural language processing was used to 
identify important topics related to COVID-19 and to attribute emotional intensity scores to comments. Local data for the Vancouver subreddit, collected between June 12, 2020 to May 21 2021, was used for the purposes of this study. A total of 7 topics were identified from Reddit comments. The majority of topics expressed negative sentiments, whereas vaccine related discussions exhibited positive sentiments. Through the identification of salient COVID-19 topics and analyzing their public sentiment trends, this can aid in the development and delivery of appropriate public health measures in Vancouver, British Columbia. A deeper understanding into the role social media has in disseminating health information and in decision making can elucidate effective ways to combat misinformation.

\section{Are You Spreading Misinformation? Analyzing How Influential Twitter Users Contribute to the Spread of COVID-19 Information}

\section{Bizhan Alatif, Jennifer Tram Su, Maggie Wang, and Sarina Xi}

When COVID-19 was declared a pandemic in early 2020, a virtual pandemic of misinformation had gone undeclared. While some of these misinformed claims may be tame, others could lead to the loss of life (e.g. Hydroxychloroquine curing COVID-19) and behavioral changes with larger ramifications (e.g. Bulk buying hand sanitizer). Therefore, detecting and removing instances of misinformation could reduce the consequences that arise from a misled public. In particular, misinformation tends to pervade social media platforms. Its convenience allows users to easily consume and spread information to others through posting. As a result of this accessibility, many have turned to these outlets as their primary source of news [1]. In 2020, the three most used social media platforms were Facebook, YouTube, and Twitter. While Facebook dominates as the go-to platform for general use, over half of Twitter's user base ( 59\%) is reliant on the platform for news [2]. Furthermore, Twitter is known as the platform that houses the personal thoughts of many wellknown figures. As opposed to Facebook or YouTube, where these interactions are scarce, users can directly engage with these influencers and easily pass on the content produced by these figures for the user's own network to see. Thus, Twitter's social structure allows information to easily propagate between influencer and user, and amongst users themselves. Accordingly, this paper examines the correlation between influ- ential Twitter accounts - right and left-leaning news sources, health organizations, celebrities, and right and left-wing politicians - and the spread of misinformed claims on Twitter. Being higher-level nodes in their social networks, these Twitter accounts are selected as their large follower counts allow for information to easily disseminate to a wider audience [3]. Visualizing these interactions can provide insight into the patterns and trends allowing for the identification and cessation of misinformation spread. This paper concentrates on misinformation, predominantly in the USA, during the early stages of COVID-19 by comparing tweets from well- known Twitter accounts to datasets consisting of "fake" and "true" claim tweets. Additionally, engagement metrics are used to gauge how much traction tweets from different influential Twitter accounts garner. With this, timelines of tweet frequency and engagement are used to determine the correlations, via application of the Kendall Rank Correlation Coefficient and Mann-Kendall significance test, between influential Twitter accounts and general fake and true tweets. The results show that the most statistically significant relationship found is between the distribution of real tweets and average likes and retweets from health organizations. Other social network nodes do not have concrete relationships with fake and real tweets.

\section{The Month the World Stood Still: An Infodemio- logical Analysis of COVID-19 Related Tweets From March 2020}

\section{Kobi Nauenberg, Shimon Nauenberg, and Ryan Ripsman}

The COVID-19 pandemic has been one of the greatest public health challenges of this generation. In order to combat the virus, public health officials in many jurisdictions have been thrust into the public eye to help educate the populace about the virus. Because of the magnitude of COVID-19's disruption and the con- stantly changing science surrounding COVID- 19, many people have lost confidence in both their governments and their public health officials over the course of the pandemic. This loss of faith has been exacerbated by the constant stream of misinformation which is spread through word of mouth and social media. In this study, we 
examined the effects of public health announcements on the spread of misinformation and the sentiment towards government and public health leaders and institutions, using Twitter data from March 2020. We found that public health announcements were followed by a spike in the spread of misinformation. However, we did not find a significant change in sentiment towards government and public health officials and organizations. This study suggests that public health officials should weigh the potential increase in the spread of misinformation against the potential benefits before making a public health announcement.

\section{COVID-19 Vaccination Rates in India: A Senti- ment Analysis of the Impact of the Farmers' Protest}

\section{Michelle Lam and Aleisha Fernandes}

The COVID-19 pandemic has brought forth a number of social movements including the Farmers' Protests in India. However, there is a lack of information available about the implications of social movements on vaccination rates as well as on new infections and mortality rates. With Twitter being a popular place for people to post life updates and an excellent source of data, a sentiment analysis of tweets concerning vaccines was conducted for before and during the Farmers' Protest. Additional analyses looked at trends between vaccine tweets and the vaccination rate in India, as well as a sentiment analysis of tweets concerning both the Farmers' Protest and vaccinations. Results showed that there was a small positive linear association between vaccination tweets and vaccination rate. However, there was no statistically significant change found for vaccine sentiments. Future analysis is needed to determine if these results will differ as the protest goes on and the current situation in India continues. Additional analysis should also look at the implications misinformation plays on vaccine sentiments. 